www.nature.com/hr

\title{
Asymmetric blood pressure caused by extremely rapid cyclic fluctuations and reversible cardiomyopathy in pheochromocytoma
}

\author{
Hypertension Research (2012) 35, 1201-1202; doi:10.1038/hr.2012.121; published online 16 August 2012
}

A 56-year-old man presented to the hospital with waxing and waning headache, diaphoresis, chest distress and dyspnea for 5 days. He also experienced several brief episodes of amaurosis and falling down. The patient's history was notable for 4 years of intermittent headache, which was associated with hypertension. He was treated with antihypertensive medicine. He also had diabetes mellitus for 5 years. Initial physical examination revealed that his blood pressure was $114 / 72 \mathrm{~mm} \mathrm{Hg}$ in the right arm and 192/ $114 \mathrm{~mm} \mathrm{Hg}$ in the left arm, with a heart rate of 65 beats $\mathrm{min}^{-1}$. The ECG showed left ventricular (LV) hypertrophy with ST-segment elevation in leads V1 to V5 and extensive T-wave inversion, which was significantly different from the ECG performed 4 years previously (Figure 1). Creatine kinase was in the normal range, and troponin $T$ values were slightly increased $\left(129.5 \mathrm{pg} \mathrm{ml}^{-1}\right.$, normal values $0-100 \mathrm{pg} \mathrm{ml}^{-1}$ ). The echocardiogram showed mild increased LV wall thickness and hypokinesia of the antero-lateral segments, as well as decreased overall systolic function with an estimated ejection fraction (EF) of $41 \%$. Based on the clinical presentation and initial findings, the final diagnosis was unclear, but acute myocardial infarction and aortic dissection were considered the two most likely differential diagnoses. The patient was subsequently referred for a contrast-enhanced CT for presumed aortic dissection, which revealed a normal aorta. In addition, a large $5 \times 4 \mathrm{~cm}$ incidental mass with heterogeneous enhancement was identified in the left adrenal gland, suggesting pheochromocytoma. This finding raised the possibility that the asymmetric blood pressure in the arms that we observed at admission may have been due to extremely rapid fluctuations of blood pressure associated with pheochromocytoma. As expected, the patient's blood pressure rapidly changed within few seconds.

The patient then underwent emergent cardiac catheterization, which showed normal coronary arteries but significant hypokinesia of the antero-lateral segments and impairment of LV function (LVEF 51\%) (Figure 2a). During the cardiac catheterization, his blood pressure showed rapid increases in systolic blood pressure, up to $248 \mathrm{~mm} \mathrm{Hg}$, and then decreased as low as $77 \mathrm{~mm} \mathrm{Hg}$ every $1-2 \mathrm{~min}$ in a recurring pattern (Figure 2b). Urinary vanillyl mandelic acid levels were evaluated and found to be increased $(103.56 \mu \mathrm{mol}$ per $24 \mathrm{~h})$. The diagnosis of pheochromocytoma was therefore confirmed. The patient's condition stabilized with general supportive management, oral prazosin and fluid repletion. The marked periodic fluctuation of blood pressure decreased over time and disappeared within 1 week. The patient then underwent surgical resection of the adrenal mass. The histological examination confirmed the diagnosis of pheochromocytoma. Echocardiography performed before discharge revealed normal LV systolic function, as well as an absence of segmental wall motion abnormality. The ECG showed recovery of ST-segment elevation and normalization of the inverted $\mathrm{T}$ wave (Figure 1). The final diagnosis was that of reversible cardiomyopathy precipitated by pheochromocytoma crisis.

One of the prominent clinical features of this case is the marked reversibility of cardiomyopathy induced by pheochromocytoma. There is a resemblance to stress-induced cardiomyopathy, also known as tako-tsubo

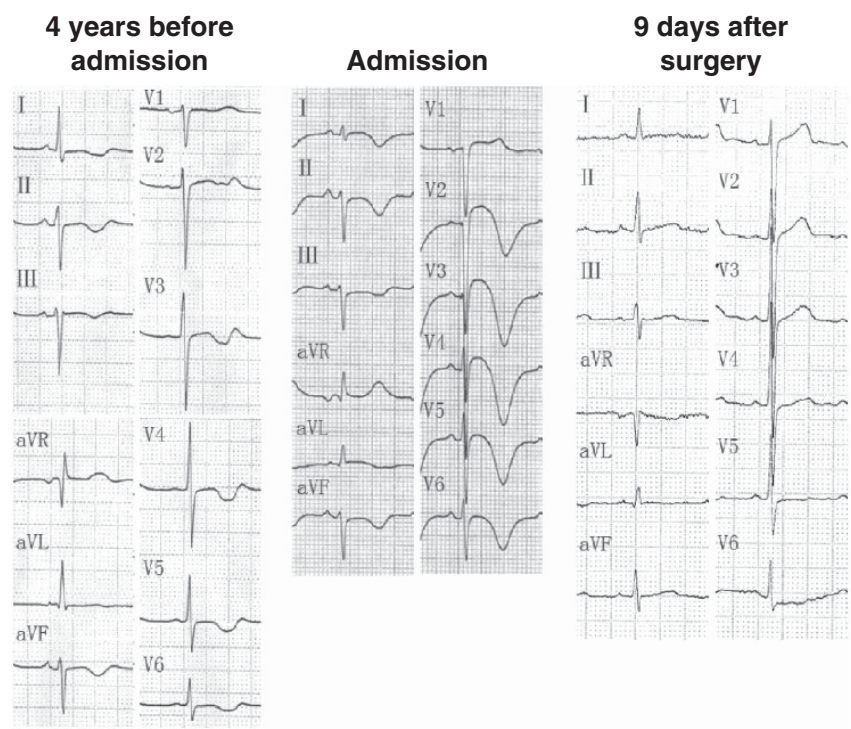

Figure 1 The time-course of the ECGs. The ECGs were performed at $10 \mathrm{~mm} \mathrm{mV}^{-1}$ and $25 \mathrm{mms}^{-1}$, with the exception that leads V1 to V6 in the ECG before admission were performed at $5 \mathrm{~mm} \mathrm{mV}^{-1}$. 

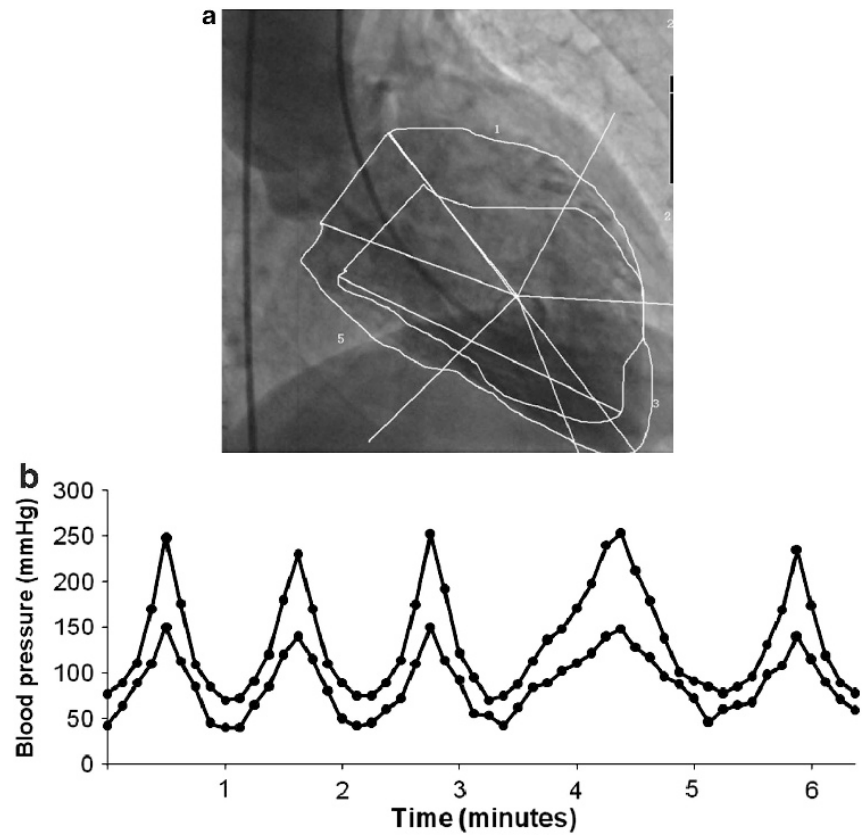

Figure 2 (a) Left ventriculogram at end-diastole and end-systole showing significant hypokinesia of the antero-lateral segments. (b) Cyclic fluctuations of systolic and diastolic blood pressure before therapy.

cardiomyopathy, which is a well-described entity characterized by a typical contractile abnormality consisting of apical and midventricular akinesis or dyskinesis and hyperkinesis of the base. However, there is no unique ventricular dysfunction pattern in pheochromocytoma-related cardiomyopathy because case reports of patients with lesions involving areas other than the apex have been reported. ${ }^{1,2}$ In the present case, the patient had midventricular hypokinesia without typical apical ballooning. Moreover, we observed pronounced ECG changes in the patient: from prominent ST-segment deviation and T-wave inversion on admission, mimicking an acute coronary syndrome, to a normal ECG on recovery within 10 days of surgery. The postulated mechanisms underlying the association between sympathetic abundance and myocardial stunning include multivessel epicardial spasms, microvascular spasms and direct catecholamine-mediated myocyte injury.

Another feature of this case was the extremely rapid cyclic fluctuation of blood pressure, with each complete cycle lasting around 1-2 min. Although paroxysmal hypertension in pheochromocytoma is well recognized, such episodes occur at irregular and often unpredictable intervals. The type of periodic changes seen in our patient is unusual. There are case reports describing alternating cycles of hypertension and hypotension as a manifestation of pheochromocytoma. ${ }^{3,4}$ To our knowledge, the short cyclic intervals seen in our case have not been reported before. A similar case reported by Otsuka et al. ${ }^{5}$ showed a periodic fluctuation of blood pressure with cyclic changes at 20 min intervals and transient LV apical ballooning in pheochromocytoma. The precise mechanisms for these alternating attacks are unclear, but the attacks are probably caused by a reflex mechanism involving either chemoreceptors or baroreceptors, or an ischemic response of central nervous system, together with hypovolemia. The infusion of fluid and alpha-adrenergic blockade could terminate these cyclic blood pressure changes.

Xiao-Yan Zheng, Dao-Quan Peng, Shui-Ping Zhao and Shu Duan

Departments of Cardiology, the Second Xiangya Hospital, Central South University, Changsha, China E-mail: pengdq@hotmail.com orzhaosp@medmail.com.cn

1 Verrijcken A, Sciot R, Dubois CL. From trivial headache to life-threatening disease. Int J Cardiol 2011; 146: e7-e9.

2 Sanchez-Recalde A, Costero O, Oliver JM, Iborra C, Ruiz E, Sobrino JA. Images in cardiovascular medicine. Pheochromocytoma-related cardiomyopathy: inverted takotsubo contractile pattern. Circulation 2006; 113 e738-e739.

3 Kobal SL, Paran E, Jamali A, Mizrahi S, Siegel RJ, Leor J. Pheochromocytoma: cyclic attacks of hypertension alternating with hypotension. Nat Clin Pract Cardiovasc Med 2008; 5: 53-57.

4 Ganguly A, Grim CE, Weinberger MH, Henry DP. Rapid cyclic fluctuations of blood pressure associated with an adrenal pheochromocytoma. Hypertension 1984; 6 : 281-284.

5 Otsuka M, Kohno K, Itoh A. Periodic fluctuation of blood pressure and transient left ventricular apical ballooning in pheochromocytoma. Heart 2006; 92. 1837. 